\title{
WAS BLEIBT? ZUM FORTLEBEN DER DDR-LITERATUR IN DER FORSCHUNG
}

\author{
ABSTRACT. What remains? The afterlife of GDR literature in the research
}

The title of the story What Remains by Christa Wolf denotes one of the main topics of the present discussion about German literature after 1989. The article presents the new questions (e.g. one or two German literature(s)? What does 'GDR literature' mean? Is it a 'special case'?) and changing conditions of the study of East German literature. A few new historical works discussing German literature since 1945, mainly since the reunification, and a few theoretical approaches (e.g. GDR literature as a chronotope and as regional literature) are presented.

KEY WORDS: contemporary history of German literature, GDR literature, post-GDR literature, reunification, memory culture, 'master narrative'

Die Veröffentlichung der Erzählung Was bleibt von Christa Wolf (1990) löste eine Serie von Debatten aus, die in der Literaturforschung mit dem Sammelbegriff „deutsch-deutscher Literaturstreit“ benannt wurde (Grub, 2009 mit weiteren Literaturhinweisen). In der öffentlich ausgetragenen Auseinandersetzung handelte es sich nicht nur um Christa Wolf, ihren schriftstellerischen Rang und die (vermeintlich mangelnde) Rechtschaffenheit, sondern auch um den Wert der DDR-Literatur, ihre moralische Legitimierung und die Frage, was von ihr überhaupt bleiben wird. Die Wolf-Debatte kann aus heutiger Perspektive nicht zuletzt als „Chiffre eines grundlegenden Diskurswandels“ gelesen werden, in dem „es um die Abwicklung des gesamten kulturellen Systems DDR“ ging (Reimann, 2008, S. 11). Dieser Prozess ist wohl in einem breiteren erinnerungskulturellen Kontext zu sehen, in dem die Aufarbeitung der DDR-Geschichte erfolgt, nicht zuletzt als die Erinnerung an die zweite deutsche Diktatur (Assmann, 2013). Der Literaturstreit um Christa Wolf entwickelte sich zu einer Grundsatzdebatte, in der „das althergebrachte politische Koordinatensystem des nachkriegsdeutschen Literaturbetriebs ins Wanken [geriet]. Und mit ihm die ästhetische Beurteilung der zwischen 1949 und 1989 verfaßten gesamtdeutschen 
Literatur" (Weininger, 2004, S. 133). Den weiteren Kontext für den deutsch-deutschen Literaturstreit bildet die politisch determinierte Aufarbeitung der SBZ/DDR-Vergangenheit mit den Schwerpunkten Delegitimation der DDR, Etablierung einer gesamtdeutschen demokratischen Erinnerungskultur und Renationalisierung des Gedenkens „mit wieder verschärfter kommunismuskritischer und antikommunistischer Stoßrichtung" (Eke, 2013, S. 15).

Der Status der DDR-Literatur, die noch in den 1970er und 1980er Jahren außerhalb des Landes Anerkennung und breite Leserschaft fand, veränderte sich nach der Wende völlig. In der unmittelbaren Nachwendezeit wurde selbst den in der BRD hochgeschätzten Autoren wie Christa Wolf, Heiner Müller, Stephan Hermlin, Stefan Heym, Volker Braun und Christoph Hein vorgeworfen, sie hätten „ein verfehlte[s] Sinngebungsprojekt der Geschichte" moralisch legitimiert (Eke, 2013, S. 13). Repräsentativ für den neuen externen Blick auf die DDR-Literatur ist das 1992 formulierte Urteil von Thomas Schmidt über „das Elend dieser feinziselierten Literatur, dieser oft genug verquasten Innerlichkeit, dieser ebenso großmäuligen wie öden Brecht-Nachfolge“, die „ohne den schützenden Kokon des Staates, in dem sie entstanden war, augenblicklich unreif, bramarbasierend, kitschig und wie Schnee von gestern wirkt." (Schmidt, 1992, S. 175, zit. nach Eke, 2013, S. 14) Unabhängig von solchen scharfen Urteilen, in denen die westdeutsche Kritik zu keineswegs wertneutralen Begriffen wie „Gesinnungsästhetik“, „Gesinnungskitsch“ oder „Stillhalteliteratur" griff (Emmerich, 1998, S. 146), wurde nach der Wende das Postulat einer kritischen Re-Lektüre der bisher vorhandenen Entwürfe und Konzepte der ost- und westdeutschen Literatur gestellt. Die Ansätze dieser Re-Lektüre, die im Folgenden überblickend dargestellt werden, enthüllen nicht zuletzt Defizite der Forschung, in der kaum neue übergreifende Ansätze und Konzepte herausgearbeitet werden. Trotz einer kaum überschaubaren Menge von Einzelstudien und -untersuchungen zu bestimmten Autoren und verschiedenen Aspekten der ostdeutschen Literatur, gibt es bis heute keine völlig neue Gesamtdarstellung der deutschsprachigen Literatur. Die Unsicherheit der Literaturforschung im Umgang mit der DDR-Literatur äußert sich in eher diffusen Antworten auf drei - so Wolfgang Emmerich - „Gretchen-Fragen“ nach Art und Zahl der deutschen Literaturen seit 1945: 1. Wie viele deutsche Literaturen gibt es? 2. Sollen sie gemeinsam oder getrennt behandelt werden? 3. Wie viele DDR-Literaturen gibt es? (Emmerich, 2013, S. 49). Der Schriftsteller Erich Loest sprach bereits 1984 von vier DDR-Literaturen. Ihre Repräsentanten seien systemkonforme, systemkritische Autoren, Autoren, die in der DDR leben, aber nur in der BRD publiziert werden sowie emigrierte Autoren, die in der BRD über die DDR schreiben (Loest, 1984). Die im Zuge der deutsch-deutschen Literaturdebatte formulierte These von Walter Jens: „Es gab zwei deutsche Staaten, aber es gab nur eine deutsche Literatur" (Jens, 1990) wurde in der Literaturforschung bis heute weder bestätigt noch eindeutig widerlegt. 


\section{Was ist „DDR-Literatur"?}

Probleme der Literaturwissenschaftler mit der Literatur, die in der DDR entstand, beginnen bereits bei der Verwendung des Begriffs. Ist sie als „DDR-Literatur“, „Literatur in der DDR“, „Literatur aus der DDR“, „Literatur über die DDR“, oder - im Hinblick auf ihre mögliche Nachgeschichte - als „Literatur nach der DDR“, „PostStaatliche DDR-Literatur" bzw. „Post-DDR-Literatur“ zu bezeichnen? (Ludwig \& Meuser, 2009; zum Begriff der „Post-Staatlichen“ bzw. der „Post-DDR-Literatur“ Galli, 2013). Was wird mit diesen Begriffen benannt: politisch konforme Literatur, Dissidenten-Literatur, Literatur zwischen Affirmation und Ablehnung, Literatur, die eigenen künstlerischen und ästhetischen Prinzipien folgte und (zugleich) reformsozialistische Hoffnungen und Illusionen zum Ausdruck brachte? (Emmerich, 1998, S. 159). Die in den aufgezeigten Begriffsvarianten und Schwerpunktsetzungen anklingende Vorstellung von der Eigenständigkeit des literarischen Forschungsfeldes und seiner Homogenität war und ist in der Forschung alles andere als selbstverständlich.

Betrachtet man den Status der DDR-Literatur in der Bundesrepublik in einer literaturhistorischen Perspektive, so kann man eine Entwicklungslinie von der (fast) Nichtbeachtung in den 1950er Jahren bis hin zur Anerkennung ihres moralischen und ästhetischen Ranges in den späten 1970er und einer insgesamt positiven Beurteilung in den 1980er Jahren zeichnen (Opitz, 2009; Hofmann, 2009; Ludwig, 2013 mit weiterführenden Literaturhinweisen). Die westdeutsche Rezeption der DDR-Literatur hatte in den 1950er Jahren einen entschieden politischen Charakter; die ostdeutsche Literatur wurde - bis auf wenige Ausnahmen (u. a. Peter Huchel) als kommunistische Propaganda angesehen und daher für künstlerisch wertlos erklärt. Von Bertolt Brecht, Anna Seghers oder Arnold Zweig wurde damals kaum Notiz genommen.

In der westdeutschen Literaturforschung der 1960er Jahre setzte sich die Wahrnehmung der DDR-Literatur als eines selbständigen Phänomens durch. Diese Literatur, die „zu einer Art landeskundlichem Reservoir für Westdeutsche [wurde]“ (Emmerich, 1998, S. 143), machte zugleich durch ästhetische Qualitäten der Lyrik und Prosa von Johannes Bobrowski, der Romane u. a. von Uwe Johnson, Christa Wolf, Günter de Bruyn, Erwin Strittmatter, der Lyrik von Günter Kunert, Wolf Biermann und der Dramen von Heiner Müller, Peter Hacks oder Volker Braun auf sich aufmerksam. In den späten 1960er Jahren bildete die ostdeutsche Literatur (die in der DDR spätestens mit der Machtübernahme Honeckers 1971 offiziell als ein eigenständiges literarisches System anerkannt wurde) ,eine[n] umrissene[n] eigene[n] Corpus" auch für die westdeutsche Öffentlichkeit, insbesondere für die Nachfolger der Studentenbewegung und für die Vertreter der Neuen Linken, die die systemkritische Literatur aus der DDR (vor allem Wolf Biermann, Volker Braun, Christa Wolf und Heiner Müller) als „Spiel-Raum, als Projektionsfläche für eigene 'utopische' 
Entwürfe einer nichtkapitalistischen Gesellschaft" betrachteten (Emmerich, 1998, S. 143). Im Zuge der in den 1970er Jahren erfolgten Öffnung der westdeutschen Literaturwissenschaft und Literaturkritik (u. a. Frank Trommler, Marcel ReichRanicki und Fritz J. Raddatz) auf die undogmatischen und systemkritischen Autoren nahm die Literaturforschung die „Konvergenzbewegungen“ zwischen ost- und westdeutscher Literatur in den Blick, die man in den 1980er Jahren in einigen parallelen Themen, wie Zivilisationskritik, Frauenbewegung, atomare Bedrohung u. a., entdeckt zu haben glaubte (Ludwig, 2013, S. 68). Dennoch aber blieb die getrennte, institutionell gesicherte Behandlung beider Literaturen bestehen. Auch manche ostdeutschen Autoren (u. a. Christoph Hein und Stefan Hermlin) akzentuierten die Trennung. Die Existenz zweier deutscher Literaturen galt insgesamt zwischen etwa 1970-1990 als eine „recht stabile Konvention“ (Ludwig, 2013, S. 69). Die DDR-Literatur war aber - so die Befürworter ihrer Eigenständigkeit - ein „heteronomes Gebilde“ (Opitz, 2009, S. 73) mit verschiedenen Funktionen, die „,von der Panegiryk bis zur Moraldidaktik reichen" (Heukenkamp, 1996, S. 31, zit. nach Opitz, 2009, S. 73). Beachtenswert ist in diesem Zusammenhang auch der Versuch, „Leitbegriffe“ eines Diskurses der DDR-Literatur als „sozialistischer Literatur“ herauszufinden, der von Peter Böthig unternommen wurde:

Geschichte als kontinuierlicher, teleologischer Prozeß; Fortschritt als prozessualer Modus der Geschichte; Utopie als sozial relevante, die Gesellschaft vorantreibende Kraft; Gesellschaft als Quelle von Sinn; antagonistisches Staats- und Klassenmodell; Subjekt als Zentrum von Texten; Literatur als (wie auch immer kritische, widersprechende, therapeutische) Repräsentation nachprüfbarer Realitäten. (Böthig, 1997, S. 11, zit. nach Ludwig, 2013, S. 75)

Im Zuge der nach 1989 entfachten Diskussion, ob man überhaupt von einer DDR-Literatur sprechen könne, versuchte man auch ihre Besonderheiten herauszuarbeiten. Den Ausgangspunkt bildete die Überzeugung, dass die DDR-Literatur unter anderen Umständen als die westdeutsche Literatur entstand und sich entwickelte. Dementsprechend hob Jurek Becker die Ersatzfunktion der ostdeutschen Literatur hervor, die - als Vertretung der fehlenden Öffentlichkeit - ,der letzte öffentliche Ort [war], an dem noch Meinungsverschiedenheiten ausgetragen wurden" (Becker, 1996, S. 120). Als differentia specifica der DDR-Literatur wird auch eine einzigartige Verschränkung von Literatur und Gesellschaft betrachtet, die in Ostdeutschland ein spezifisches Verständnis der Literatur als ,maßgebliches Instrument zur Herstellung, ja sogar Erziehung einer neuen Gesellschaft" hervorgebracht und eine ,übernatürliche Wirkungsmacht der Literatur" garantiert habe (Ludwig, 2013, S. 76). Das literarische Engagement der meisten DDR-Autoren, welches als Unterscheidungsmerkmal der DDR-Literatur nicht unumstritten ist (Ludwig, 2013, S. 79), war durch einen doppelten Druck determiniert: seitens der staatlichen Zensur und seitens des Publikums. Beides habe - so Christoph Hein in einer kritischen Selbstbetrachtung zur für die Literatur gefährlichen Politisierung und Radikalisierung geführt: 


\begin{abstract}
Man wurde von zwei Seiten bedrängt, und die Literatur war von zwei Seiten bedroht - vom staatlichen Zensor und von den Erwartungen des Publikums. Dem Druck des Staates konnte man ausweichen, der war so eindeutig und offensichtlich. [...] Dem Druck des Publikums hingegen konnte man sich kaum entziehen. Die Leser wollten hören, wie ich dem Honecker das Messer in den Leib stoße. Gefragt war nicht nur der kritisch-engagierte, sondern der extrem politische Schriftsteller. (Hein, 1990, S. 44)
\end{abstract}

Das Postulat der „Entpolitisierung“ der Literaturbetrachtung und der Auswertung der ostdeutschen Literatur anhand ihrer ästhetischen Qualitäten, welches bereits im Zuge des deutsch-deutschen Literaturstreits aufgestellt wurde und in den frühen 1990er Jahren zur Ablehnung jeder politisch engagierten Literatur führte, ist in den letzten Jahren wohl wieder erkennbar in der Tendenz, den überzeitlichen Wert der DDR-Literatur gerade in den narrativen Leistungen mancher Autoren zu suchen, wovon noch die Rede sein wird.

\title{
Das Nachleben der DDR-Literatur?
}

Eine Zwischenbilanz der DDR-Literaturgeschichtsschreibung der 1990er und 2000er Jahre lässt einige Schwerpunkte (und Defizite) dieser Forschung benennen, die keineswegs als flächendeckend und systematisch zu betrachten ist. Die Aufmerksamkeit der Forscher konzentrierte sich in einem erheblichen Teil auf die Zensur- und Überwachungsthematik (Stasi) sowie auf die Wendeproblematik. Zahlreiche Untersuchungen wurden zu bestimmten Aspekten der DDR-Literatur vorgelegt, wie u. a. Frauenliteratur, alternative Literaturszene am Prenzlauer Berg, die Literatur der späten 1940er und 1950er Jahre und die ausgebürgerten bzw. übergesiedelten ostdeutschen Autoren. Auch den daheim gebliebenen Schriftstellern und ihrem Werk wurden unzählbare Einzelstudien gewidmet. Defizitär sind dagegen - wie bereits angedeutet - Gesamtbetrachtungen des literarischen Feldes DDR und neue Forschungsansätze (Emmerich, 2013). Die überblickende Gesamtbetrachtung der DDR-Literatur basiert weitgehend auf den Literaturgeschichten, die vor 1989 entstanden sind und nach der Wende aktualisiert und erweitert wurden. Ralf Schnell charakterisiert zwar seine Geschichte der deutschsprachigen Literatur seit 1945 als den „Versuch einer weitgehenden Integration auch der DDR-Literatur in die Gesamtentwicklung der deutschsprachigen Literatur seit 1945“ (Schnell, 2003, S. IX), dennoch ist in der praktischen Ausführung die getrennte Behandlung beider Literaturen erkennbar. Als Beispiel kann hier der dem literarischen Erinnerungsdiskurs gewidmete Abschnitt dienen, in dem fast ausschließlich Texte westdeutscher Autoren, wie u. a. Günter Grass, Walter Kempowski, Martin Walser, Hans-Ulrich Treichel, W. G. Sebald, Stephan Wackwitz und Bernhard Schlink behandelt wurden. Die ostdeutschen Autoren repräsentiert allein Reinhard Jirgl. Die Geschichte der deutschsprachigen Literatur von Wolfgang Beutin vertritt (Beutin, 2008) - so Matteo Galli 
in seiner Bestandsaufnahme der DDR-Literaturgeschichtsschreibung - ein „klassisches Narrativ aller Literaturgeschichten, die sich mit der Gegenwartsliteratur befassen" (Galli, 2013, S. 110), fokussiert auf die politischen Voraussetzungen der Wende, den deutsch-deutschen Literaturstreit, die Verwicklung in die Zusammenarbeit mit der Stasi einiger ostdeutscher Schriftsteller und auf Tendenzen, Diskurse und literarische Formen, die nach wie vor für beide Literaturen relevant sind. Der Versuch, die beiden Literaturen in einem möglichst engen Zusammenhang darzustellen, liegt der Neuauflage der Geschichte der deutschen Literatur von 1945 bis zur Gegenwart von Wilfried Barner zugrunde, in der die 1980er Jahre als die Periode einer zunehmenden „Durchlässigkeit“ der Literatursysteme dargestellt wurden (Barner, 2006).

Ein anschauliches Beispiel für die Änderung der Perspektive auf die DDR-Literatur bietet Wolfgang Emmerichs Kleine Literaturgeschichte der DDR. Repräsentierte der Autor in den Ausgaben von 1981 und 1989 noch die für die damalige westdeutsche Literaturgeschichtsschreibung typische Sicht, in der - wie er (selbst-)kritisch anmerkt - das Interesse am „Experiment Sozialismus“ permanent mit dem Interesse an der „Literatur an sich“" verwechselt wurde (Emmerich, 1998, S. 152), so wollte er in der Neuausgabe von 1996 (und der von 2000, die keine wesentlichen Veränderungen aufweist) „auf dem Verhaktsein der Literatur mit Geschichte und Gesellschaft“ nicht mehr beharren und auch die ästhetische Dimension der DDR-Literatur zu ihren Rechten kommen lassen (Emmerich, 1998, S. 153). Diese Ausgabe wurde um ein der Wendezeit gewidmetes Kapitel erweitert, in dem der Zusammenbruch des Systems DDR-Literatur und der literarische Streit um einige autobiografische Texte, in denen die „ostalgische“ Literatur und die melancholische Stimmung bei manchen ostdeutschen Autoren thematisiert wurden, zur Darstellung gelangten (Emmerich, 1996).

Die nach der Wende erneut gestellte Frage nach der Existenz der DDR-Literatur und ihrer Einheitlichkeit verband sich in der Literaturforschung mit der Frage, ob die deutsche(n) Literaturgeschichte(n) umzuschreiben bzw. neu zu schreiben ist bzw. sind. In der Literaturgeschichtsschreibung wird die DDR-Literatur behandelt als eigenständiges und abgeschlossenes Phänomen (Wolfgang Emmerich, Ursula Heukenkamp), als integraler Teil der deutschen Literatur nach 1945 (Ralf Schnell), als Teilliteratur neben der westdeutschen, österreichischen und schweizerischen bzw. als Regionalliteratur (Skare, 1999). Umstritten bleibt nach die vor die Frage nach der Bedeutung der Wende als einer Zäsur in der deutschen Gegenwartsliteratur. Hat es nach 1989/90 einen radikalen Bruch gegeben, oder kann man eher von (auch personalen) Kontinuitäten sprechen (Žmegač, Škreb \& Sekulić, 1997; Bogdal, 1998)? Anders gefragt, kann man die Existenz der DDR-Literatur auch in die Nachwendezeit verlängern und die ,poststaatliche DDR-Literatur“ bzw. „Post-DDR-Literatur“ als ein autonomes Forschungsfeld betrachten? Trotz einiger Versuche, das ,literarische Nachleben der DDR" (Helbig, 2007) systematisch darzustellen, scheint selbst der Begriff der „Post-DDR-Literatur“ - so die 2010 formulierte Diagnose - weiter- 
hin ein unerfülltes Desiderat der Forschung zu sein (Galli, 2013, S. 114). Ein populäres Modell der Behandlung der „Post-DDR-Literatur“ entwickelte Astrid Köhler in ihrer Studie unter dem geradezu programmatischen Titel Brückenschläge. DDR-Autoren vor und nach der Wiedervereinigung (Köhler, 2007). Die Verfasserin konzentrierte sich auf Kontinuitäten, Parallelen und Brüche vor und nach 1989/90 im Werk u. a. von Christa Wolf, Klaus Schlesinger, Ulrich Plenzdorf, Christoph Hein und Angela Krauß. Intertextuelle Bezüge und Verweisungszusammenhänge zwischen den älteren und neueren Texten der jeweiligen Autorinnen und Autoren lassen Köhler die These von ihrer Rückkehr zu erprobten Schreibweisen (spätestens Mitte 1990er Jahre) und ihrer Arbeit an einem auch ästhetisch kohärenten Gesamtwerk formulieren (Köhler, 2007).

Eine weitgehende Kontinuität von den vor 1989 ausgeprägten ästhetischen Strategien wird auch in dem Sammelband von Janine Ludwig und Mirjam Meuser diagnostiziert, in dem die Texte von so unterschiedlichen Autorinnen und Autoren wie u. a. Stefan Heym, Stephan Hermlin, Hermann Kant, Peter Hacks, Sarah Kirsch, Klaus Schlesinger, Wolfgang Hilbig, Monika Maron, Sascha Anderson, Durs Grünbein und Reinhard Jirgl behandelt wurden (Ludwig \& Meuser, 2014).

Auf Kontinuitäten zwischen der DDR-Literatur und der „Post-DDR-Literatur“ wird auch indirekt im Lexikon von Michael Opitz und Michael Hoffmann hingewiesen. Sie haben ihren Ursprung - so die beiden Herausgeber - in den seit den 1970er Jahren gegebenen „Konvergenzen“ der Literatur in den beiden deutschen Staaten, die unter den Stichworten Friedens- und Ökologiebewegung, allgemeine Zivilisationskritik, Literatur von Frauen subsumiert werden können (Hofmann, Opitz \& Kanning, 2009, S. VI). Unter den dort behandelten Autoren befinden sich sowohl die „Klassiker“ der späten DDR-Literatur (u. a. Christa Wolf, Christoph Hein, Volker Braun, Günter de Bruyn, Erich Loest, Wolfgang Hilbig und Monika Maron) als auch Debütanten, die ihre ersten Werke in den 1990er bzw. 2000er Jahren publizierten (u. a. Thomas Brussig, Julia Franck, Annett Gröschner, Ingo Schulze, Reinhard Jirgl, Lutz Seiler, Jana Hensel, Uwe Tellkamp und Antje Rávic Strubel).

Die mit dem Sammelbegriff „DDR-Schriftsteller“ bezeichneten Autoren bilden keine generationelle Einheit. Diese Erkenntnis liegt den Versuchen zugrunde, die Autorenlandschaft in der DDR generationsspezifisch zu strukturieren, wie in Emmerichs Kleine Literaturgeschichte der DDR. Die älteste Generation, die den Nationalsozialismus und den Krieg erlebte, den antifaschistischen Gründungsmythos der DDR übernahm und den deutschen „Arbeiter- und Bauernstaat“ aufgebaut hat, repräsentiert in Emmerichs Generationsmodell Christa Wolf. Zu der mittleren Generation der Ende der 1930er bis 1950er Jahre Geborenen, die an dem Aufbau des Sozialismus willig, wenn auch nicht unkritisch teilnahm, gehören in seiner Darstellung u. a. Helga Königsdorf, Brigitte Burmeister oder Thomas Rosenlöcher. Die jüngste Generation der sogenannten Hineingeborenen in das System der DDR, die aber zugleich das Nicht-involviert-Sein in das Literatursystem DDR kennzeichnet, vertreten 
Kerstin Hensel und Thomas Brussig. Es gibt auch Versuche, diese Generationsschichtung zu verfeinern bzw. zu erweitern und diejenigen Autoren mit einzubeziehen, die - obwohl sie noch in der DDR sozialisiert wurden (wie z. B. Ingo Schulze, Jana Hensel und Antje Rávic Strubel) - ihre Karrieren erst nach der Vereinigung begonnen haben. Die generationsorientierte Reflexion der Forschung, auch der soziologischen Forschung (Ahbe \& Gries, 2006), begleitet die Suche nach übergreifenden Gemeinsamkeiten, die es erlauben, die DDR-Autoren als eine, wenn auch nicht homogene, Gruppe zu betrachten. So wird der spezifische Autorenstatus, die Erfahrung mit zwei verschiedenen Gesellschafts- und politischen Systemen, das Verständnis von Literatur als Form öffentlicher Kommunikation, die Neigung zur Reflexion über die Geschichte und über den Einfluss der Ideologie auf alle gesellschaftlichen Bereiche als kennzeichnend genannt (Köhler, 2007, S. 213-229). In die Betrachtung werden auch die aus der DDR ausgereisten bzw. ausgewiesenen Schriftsteller einbezogen, wodurch die Existenz der DDR-Literatur als Produkt der literarisch engagierten Autoren über die geografischen und zeitlichen Grenzen des ostdeutschen Staates hinaus verlängert wird (Ludwig \& Meuser, 2009; Ludwig \& Meuser, 2014).

\section{Die Re-Lektüre der deutschen Literatur?}

Das Postulat der Re-Lektüre der DDR-Literatur nach 1989 ist mit dem Wunsch verbunden, auch die bisherige Forschung, die sich in den einschlägigen Gesamtdarstellungen vor 1989 auf das gesellschaftlich-politische System und seine Evolution, die staatliche Kulturpolitik und die thematisch behandelte Literatur konzentrierte, kritisch zu hinterfragen, ohne - so Emmerich - „den 'drei alten erkenntnistheoretischen Illusionen“ der Einfachheit, Ganzheit und Substantialität zu verfallen“ (Emmerich, 2013, S. 157; zum Fortbestehen des tradierten Musters in der Erforschung der DDR-Literatur siehe Köhler, 2007, S. 14). In den zehn Jahre nach der Wende formulierten Postulaten einer neuen Lesart der DDR-Literatur ging es vornehmlich um Entpolitisierung der Literaturbetrachtung:

Beruht ein anderes Verständnis von fiktionalen Texten, die vor 1989 geschrieben wurden, nach 1989 lediglich auf neuen Informationen, welche wiederum die Grundlage für neue Analysen oder Interpretationen bieten? Oder sind die neuen Lesarten das Produkt einer grundlegenden politischen Umorientierung? Anders ausgedrückt: Dreht es sich um eine Horizonterweiterung im Sinne der Hermeneutik, oder unterwerfen die neuen Machtverhältnisse den Diskurs der DDR-Literatur einer politisch motivierten Umwertung? (Hoppe, 1999, S. 45)

Die Ende der 1990er Jahre postulierte Re-Lektüre der DDR-Literatur umfasste auch eine komparative Herausarbeitung der Parallelen zwischen Sozialismus und Faschismus im Hinblick u. a. auf ästhetische Ähnlichkeiten von Stalinismus und 
Faschismus, die Determinierung des Individuums durch die Geschichte, gemeinsame Klischees und Vorurteile in der sog. sozialistischen und faschistischen Literatur und das Widerstandspotential der Literatur in totalitären Systemen (Hoppe, 1999, S. 62).

Mit dem wachsenden zeitlichen Abstand von dem Mauerfall und dem Ableben der DDR wird eine solche „Horizonterweiterung“ zunehmend in Angriff genommen, wie in dem bereits erwähnten Projekt einer (Wieder-)Annäherung an die DDR-Literatur, dessen Schwerpunkte u.a. literaturgeschichtliche Wertungsfragen, Gattungsentwicklung, ästhetische und politische Kontexte der frühen SBZ/DDRZeit, die späte DDR- und die Wendezeit-Literatur bilden (Eke (Hrsg.), 2013). Hinter dem Postulat einer kritischen Re-Lektüre der DDR-Literatur (und auch der BRD-Literatur) steht die Absicht, eine systematisierende Geschichtsschreibung der ästhetischen Formen innerhalb der deutschen Literatur seit 1945 zu entfalten, der „Konstruktionsleistung von Erinnerung und Gedächtnis, an der die Medien der Repräsentation (Historiographie, Literatur, Film, Denkmäler, Gedenktage usw.) mit ihren je spezifischen Ausdrucksweisen stets 'mitschreiben'", gerecht zu werden und beide deutsche Literaturen als „Medien der Aushandlung von symbolischer Differenz in ihrer je eigenen Gegenwärtigkeit zu bestimmen“ (Eke, 2013, S. 23).

In der neuesten Forschung zur DDR-Literatur scheint die Tendenz sich durchzusetzen, sie als Teil der deutschen Literatur nach 1945 und doch als eine eigenständige literarische Epoche zu betrachten. Wolfgang Emmerich z. B. versucht mit Hilfe seines u. a. durch Bachtin, Bourdieu und das Paradigma des spatial turn inspirierten Ansatzes „den stabilen Chronotopos DDR / DDR-Literatur“ zur systematisierenden Darstellung zu bringen (Emmerich, 2013, S. 60). In den Antworten auf die Frage, was der bleibende Wert der DDR-Literatur sei, wird hauptsächlich auf zwei Aspekte hingewiesen: ihren dokumentierenden Charakter und - was damit aufs engste zusammenhängt - ihr sprachkritisches Potential. Überleben werde daher Literatur, „die Auskunft über die gesellschaftlichen Verhältnisse gibt, in denen sie entstanden ist", „in der ein inzwischen untergegangenes Staatswesen seinen Abdruck hinterlassen hat" (Opitz, 2013, S. 89, 94). Will man die DDR-Literatur als ein Archiv verstehen, so sind die in ihm aufbewahrten Bestände auf ihren Wahrheitsgehalt hin zu prüfen, ohne den ästhetischen, politischen, ethischen und anderen Wertungskriterien Vorzug zu geben: „Deshalb sollten die Werke von Hermann Kant nicht unter den Tisch fallen, auf dem die von Heiner Müller liegen“ (Opitz, 2013, S. 95; zu der neuesten material- und archivorientierten Erforschung der DDR-Literatur vgl. u. a. Bülow, Wolf \& Neumann, 2014). In dieser Sicht verdienen die Werke von Erik Neutsch oder Walter Werner gleichermaßen das Interesse der Literaturwissenschaft wie die von Johannes Bobrowski, weil sie - die kritisch zu prüfende - Auskunft geben über Art und Weise der Auseinandersetzung mit der Geschichte bzw. der Aufarbeitung der Vergangenheit. Die Herausarbeitung einer gemeinsamen ästhetisch-formalen Basis der DDR-Literatur scheint durchaus problematisch, wenn man von gewissen gemeinsamen Merkmalen absieht, wie eine Neigung zum realistischen Erzählen und 
ein stark ausgeprägtes Formbewusstsein in der Prosa und einer spezifischen Rezeption des klassischen Erbes in Lyrik und Drama. Entscheidend für den epochalen Charakter der DDR-Literatur scheint - so Janine Ludwig - ein „fortlaufender Diskurs über das Selbstverständnis sozialistischer Literatur" zu sein, an dem in den 1980er Jahren selbst die Dissidenten, wie u. a. Christoph Hein und Rainer Kirsch, teilgenommen haben (Ludwig, 2013, S. 82). Ihre oppositionelle Haltung, der 'Widerstand in der Sprache' (z. B. von Wolfgang Hilbig und Reinhard Jirgl), artikuliert sich nicht zuletzt in einer spezifischen Sprachverwendung, die - so Jirgl - notwendig ist, um die auch thematisch eigenartige Literatur nicht vergessen zu lassen:

Literatur, die sich in die Geschichte versenkt, die die Vergangenheit umgräbt, die nach möglichen Ursachen für geschichtliche Verwerfungen sucht und sich nicht mit Beschwichtigungen zufrieden gibt, wird schwer in Vergessenheit geraten, wenn sie für diese Themen eine überzeugende Sprache gefunden hat. (Jirgl, 2008, S. 109-110)

Diese „,̈berzeugende Sprache“ - so noch einmal Jirgl - muss imstande sein, „die Spannungen und Konflikte der äußeren Wirklichkeit“" in „bearbeiteter, zugespitzter, 'inszenierter' Wirklichkeits-Form" in die im Text dargestellte Wirklichkeit zu bringen (Jirgl, 2008, S. 109-110). Diese Sprache, wie immer sie geartet sein mag, muss die bisher benutzten und ausgedienten Erzählmuster ersetzen.

\section{Die DDR als Erinnerungsort}

Die gegenwärtige Re-Lektüre der deutsch-deutschen Literatur beruht weitgehend auf erinnerungsgeschichtlichen und erinnerungskulturellen Konzepten, in den es nicht mehr um eine Rekonstruktion der Vergangenheit geht, sondern um die Konstruktionsleistung von Erinnerung und Gedächtnis, an der Medien der Repräsentation (Geschichtschreibung, Literatur, Film, Museumswesen u. a.) beteiligt sind (Assmann, 2007). In den Fokus des Interesses rückt dabei die „wechselseitige Durchdringung von Narrativen, Erinnerungen und kulturellen Identitäten" (GoudinSteinmann, 2013, S. 15). In Anlehnung an kultur- und erzähltheoretische Ansätze von u. a. Aleida Assmann, Ansgar Nünning und Paul Riœur sucht man nach einer ostdeutschen Erinnerungskultur und ihrer spezifischen Sprache:

Gibt es also eine spezifisch ostdeutsche Erinnerungskultur, die sich in einigen für die neuen Länder typischen Erzählungen (und auch in einer noch spezifischen Sprache) widerspiegelt? Was ist von dem Leben in der DDR als kollektive Erfahrung übrig geblieben, und wie wird es dann erzählt? Kann man behaupten, dass diese Erzählungen die historische Erfahrung von den konkreten Bedingungen ihres Entstehens ablösen? Wie eignen sich die Ostdeutschen die Inhalte ihres 'kulturellen Gedächtnisses' an, um sich mit ihnen auseinander zu setzen und sie zu einem Element ihrer Identität zu machen [...]? (Goudin-Steinmann, 2013, S. 15) 
Das erinnerungskulturelle Forschungsparadigma findet Anwendung in Bezug auf die „Erinnerungsliteratur“, die sich mit den bisher kaum beachteten Erfahrungen und Erinnerungen beschäftigt, welche ,bislang weder in die historischen Darstellungen noch in das kollektive Gedächtnis der Gesellschaft eingegangen sind“ (Assmann, 2011, S. 217). Der Fokus liegt dabei auf der literarischen Erinnerung an das Leben in der DDR und den Mauerfall, sowie auf der Darstellung von mentalen und individual- und kollektivpsychologischen Hinterlassenschaften des Lebens in einem autoritären System. In den literarischen Texten, die man als repräsentativ für die kritische Erinnerungsliteratur zur deutschen Vereinigung betrachtet, bzw. in den Filmen vom Ende der DDR sucht man nach ,Erinnerungsmodellen', ,Poetiken der Erinnerung' bzw. ,Deutungsmustern` der Vergangenheit (Braun, 2013, S. 81-139).

Carsten Gansel, der in Anlehnung an Aleida Assmanns Konzept der „Geschichte im Gedächtnis“ seine „Rhetorik der Erinnerung“ (Gansel, 2009a) entwickelt, in der es um die Formen narrativer Inszenierung von Erinnerungen geht, behandelt die Auseinandersetzung mit der DDR und ihrem literarischen Nachlass im Kontext eines Streits um die Erinnerung nach 1989. Die Begriffe, zu denen er greift, sind Schlüsselbegriffe der Erinnerungs- und Gedächtnisforschung: individuelles, kollektives, kulturelles, kommunikatives, generationsspezifisches, Speicher- und Funktionsgedächtnis (Gansel, 2007; Gansel, 2009b). Anhand von literarischen Texten kann das kollektive Gedächtnis in der DDR rekonstruiert werden; indem die Texte auf die Art und Weise der narrativen Inszenierung von Erinnerung (und die formalästhetische Struktur der Texte) hin untersucht werden, kann man aus ihnen etwas über die gesellschaftlichen Prozesse der Gedächtnisbildung in dem „realsozialistischen“ Staat DDR und über die dabei verwendeten „Tricks der Erinnerung“ (Uwe Johnson) erfahren. Durch „Figurationen“ und „Konfliktkonstellationen“, die Literatur erzeugt, gewinnt man Zugang zu den memorierten, verallgemeinerten, vereinheitlichten und tradierten Geschichten, welche gemeinschaftsbildend wirken (Gansel \& Zimniak, 2010, S. 14).

Identitätssuche, Geschichtsbewusstsein sowie narrative Inszenierung und erzählerische Vermittlung von Gedächtnis in der Nachwendezeit gehören zu den Themen, die in der gegenwärtigen DDR-Literaturforschung einen exponierten Platz einnehmen. Sie scheint als Teil der fächerübergreifenden erinnerungskulturellen Forschung eine längere Zukunft vor sich zu haben. Diese Vermutung ist umso wahrscheinlicher, als die Erinnerung an die DDR durchaus kontrovers ist und immer noch in den Anfängen steckt (Assmann, 2013, S. 118). Die deutsche Geschichtswissenschaft (und natürlich auch die deutsche Literaturwissenschaft) stehen vor der Aufgabe, für das vereinte Deutschland eine neue „Meistererzählung“, also „eine kohärente, mit einer eindeutigen Perspektive ausgestattete Geschichtsdarstellung" zu konstruieren, „,deren Prägekraft schulbildend wirkt - im Fach Geschichte, aber auch in den öffentlichen Diskussion und schließlich im Schulbuch“ (Sabrow, 2014, S. 147). Eine 
solche Darstellung der (Post-)DDR-Literatur, nicht zuletzt als eines Mediums der Gedächtnisbildung mit einem spezifischen erinnerungskulturellen Leistungsvermögen, ist noch nicht in Sicht.

\section{Bibliographie}

Ahbe, Th. \& Gries, R. (2006). Gesellschaftsgeschichte als Generationengeschichte. In A. Schüle, Th. Ahbe \& R. Gries (Hrsg.), Die DDR aus generationsgeschichtlicher Perspektive. Eine Inventur (S. 486-571). Leipzig: Universitätsverlag.

Assmann, A. (2007). Geschichte im Gedächtnis. Von der individuellen Erfahrung zur öffentlichen Inszenierung. München: Beck.

Assmann, A. (2011). Wem gehört die Geschichte? Fakten und Fiktionen in der neueren deutschen Erinnerungsliteratur. Internationales Archiv für Sozialgeschichte der deutschen Literatur (IASL), H. 1, 213-225.

Assmann, A. (2013). Das neue Unbehagen an der Erinnerung. Eine Intervention (S. 109-123). München: Beck.

Barner, W. (Hrsg.) (2006). Geschichte der deutschen Literatur von 1945 bis zur Gegenwart. (2. akt. u. erw. Aufl.). München: Beck.

Becker, J. (1996). Die Wiedervereinigung der deutschen Literatur. In J. Becker, Ende des Größenwahns. Aufsätze, Vorträge. (S. 118-135). Frankfurt a.M.: Suhrkamp.

Beutin, W. (Hrsg.) (2008). Geschichte der deutschsprachigen Literatur. (7. überarb. Aufl.). Stuttgart: Metzler.

Bogdal, K.-M. (1998). Klimawechsel. Eine kleine Meteorologie der Gegenwartsliteratur. In Böthig, P. (1997). Grammatik einer Landschaft. Literatur aus der DDR in den 80er Jahren. Berlin: Lukas.

Braun, M. (2013). Zwischen „Ostalgie“ und Entmythologisierung: Die DDR in der Erinnerungskultur. In M. Braun, Wem gehört die Geschichte? Erinnerungskultur in Literatur und Film (S. 81-139). Münster: Aschendorff.

Bülow, U. v., Wolf, S. \& Neumann, H. (Hrsg.) (2014). DDR-Literatur. Eine Archivexpedition. Berlin: Links.

Eke, N. O. (Hrsg.) (2013). „Nach der Mauer der Abgrund"? (Wieder-)Annäherungen an die DDR-Literatur (= Amsterdamer Beiträge zur neueren Germanistik 83). Amsterdam: Rodopi.

Eke, N. O. (2013). „Nach der Mauer der Abgrund“? (Wieder-)Annäherungen an die DDR-Literatur. In N. O. Eke (Hrsg.), ,Nach der Mauer der Abgrund“? (Wieder-)Annäherungen an die DDR-Literatur (= Amsterdamer Beiträge zur neueren Germanistik 83). Amsterdam: Rodopi.

Emmerich, W. (1996). Kleine Literaturgeschichte der DDR (S. 435-526). Leipzig: Kiepenheuer.

Emmerich, W. (1998). Versungen und vertan? Rückblicke auf 40 Jahre der DDR-Literatur und Geschichtsschreibung der DDR-Literatur. Oxford German Studies, H. 27, 141-168.

Emmerich, W. (2013). Zwischen Chronotopos und Drittem Raum: Wie schreibt man die Geschichte des literarischen Feldes DDR? In N. O. Eke (Hrsg.), ,, Nach der Mauer der Abgrund“? (Wieder-)Annäherungen an die DDR-Literatur (S. 43-64).

Erb, A. (Hrsg.) (1998), Baustelle Gegenwartsliteratur: die neunziger Jahre (S. 9-31). Opladen: Westdeutscher Verlag.

Galli, M. (2013). Post-Staatliche DDR-Literatur in der Literaturgeschichtsschreibung. Eine Bestandsaufnahme. In N. O. Eke (Hrsg.), ,Nach der Mauer der Abgrund“? (Wieder-)Annäherungen an die DDR-Literatur (S. 105-118). 
Gansel, C. (2007). Zwischen offiziellem Gedächtnis und Gegen-Erinnerung - Literatur und kollektives Gedächtnis in der Literatur. In C. Gansel (Hrsg.), Gedächtnis und Literatur in den „,geschlossenen Gesellschaften “ des Real-Sozialismus zwischen 1945 und 1989. (S. 13-37). Göttingen: V\&R unipress.

Gansel, C. (Hrsg.) (2009a). Rhetorik der Erinnerung. Literatur und Gedächtnis in den „geschlossenen Gesellschaften “ des Real-Sozialismus. Göttingen: V\&R unipress.

Gansel, C. (2009b). Vom „kulturellen Gedächtnis“ und der DDR. Streit um die Erinnerung nach 1989. Spiegel der Forschung, 26. Jg., Nr. 2, 40-45.

Gansel, C. \& Zimniak, P. (2010). Zum „Prinzip Erinnerung“ in der deutschsprachigen Gegenwartsliteratur nach 1989 - Vorbemerkungen. In C. Gansel \& P. Zimniak (Hrsg.), Das „Prinzip Erinnerung “ in der deutschsprachigen Gegenwartsliteratur nach 1989 (S. 11-15). Göttingen: V\&R unipress.

Goudin-Steinmann, E. (Hrsg.) (2013). Ostdeutsche Erinnerungsdiskurse nach 1989. Narrative kultureller Identität. Berlin: Frank \& Timme.

Grub, F. Th. (2009). Deutsch-deutscher Literaturstreit. In M. Opitz, M. Hoffmann (Hrsg.), Das Metzler Lexikon DDR-Literatur (S. 78-81). Stuttgart-Weimar: Metzler.

Hein, C. (1990). „Die alten Themen habe ich noch, jetzt kommen neue dazu.“ Gespräch mit Sigrid Löffler (März 1990). In C. Hein, Texte, Daten, Bilder. Hrsg. von L. Baier. (S. 37-44). Frankfurt a.M.: Luchterhand.

Helbig, H. (2007). Weiterschreiben. Zum literarischen Nachleben der DDR. In H. Helbig (Hrsg.), Weiterschreiben: zur DDR-Literatur nach dem Ende der DDR (S. 1-7). Berlin: Akademie.

Heukenkamp, U. (1996). Ortsgebundenheit. Die DDR-Literatur als Variante des Regionalismus in der deutschen Nachkriegsliteratur. Weimarer Beiträge, 42. Jg., H. 1, 30-35.

Hofmann, M. (2009). Rezeption der DDR-Literatur in der BRD. In M. Opitz, M. Hofmann (Hrsg.), Das Metzler Lexikon DDR-Literatur (S. 272-279). Stuttgart-Weimar: Metzler.

Hoppe, R. B. (1999). Im Westen nichts Neues? Beobachtungen und Überlegungen zum Umgang mit der DDR-Literatur nach 1989. (S. 45-63). In R. Skare, \& R. B. Hoppe (Hrsg.), Wendezeichen? Neue Sichtweisen auf die Literatur der DDR. Amsterdam: Rodopi.

Jens, W. (16. Juni 1990). Plädoyer gegen die Preisgabe der DDR-Kultur. Fünf Forderungen an die Intellektuellen im geeinten Deutschland. Süddeutsche Zeitung (o.S.).

Jirgl, R. (2008). Die wilde und gezähmte Schrift. Eine Arbeitsübersicht. In R. Jirgl Land und Beute. Aufsätze aus den Jahren 1996 bis 2006 (S. 92-122). München: Hanser.

Köhler, A. (2007). Einleitung. Es geht nicht (nur) um die DDR. In A. Köhler, Brückenschläge. DDR-Autoren vor und nach der Wiedervereinigung (S. 9-18). Göttingen: Vandenhoek \& Ruprecht.

Loest, E. (1984). Leipzig ist unerschöpflich. Über die 4 Arten von DDR-Literatur heute (= Paderborner Universitätsreden 2). Paderborn: Rektorat der Univ.

Ludwig, J. \& Meuser, M. (Hrsg.) (2009). Literatur ohne Land? Schreibstrategien einer DDR-Literatur im vereinten Deutschland. Mit einem Vorwort von Frank Hörnigk. Bd. 1. Freiburg: Fördergemeinschaft Wiss. Publikationen von Frauen.

Ludwig, J. \& Meuser, M. (Hrsg.) (2014). Literatur ohne Land? Schreibstrategien einer DDR-Literatur im vereinten Deutschland. Mit einem Vorwort von Jost Hermand. Bd. 2. Eschborn: Fördergemeinschaft Wiss. Publikationen von Frauen.

Ludwig, J. (2013). Was war und ist DDR-Literatur? Debatten um die Betrachtung der DDR-Literatur nach 1989. In N. O. Eke (Hrsg.), ,Nach der Mauer der Abgrund"? (Wieder-)Annäherungen an die DDR-Literatur (S. 65-82).

Hofmann, M., Opitz, M. \& Kanning, J. (Hrsg.) (2009). Metzler Lexikon DDR-Literatur. Autoren, Institutionen, Debatten. Stuttgart: Metzler.

Opitz, M. (2009). DDR-Literatur (Begriff). In M. Hofmann, M. Opitz \& J. Kanning (Hrsg.), Metzler Lexikon DDR-Literatur. Autoren, Institutionen, Debatten. (S. 72-73) Stuttgart: Metzler. 
Opitz, M. (2013). Was wird von der DDR-Literatur bleiben? In N. O. Eke (Hrsg.), „Nach der Mauer der Abgrund"? (Wieder-)Annäherungen an die DDR-Literatur (S. 83-103).

Reimann, K. E. (2008) Schreiben nach der Wende - Wende im Schreiben? Literarische Reflexionen nach 1989/90. Würzburg: Königshausen \& Neumann.

Sabrow, M. (2014). Meistererzählungen der Zeitgeschichte. In M. Sabrow, Zeitgeschichte schreiben. Von der Verständigung über die Vergangenheit in der Gegenwart (S. 147-159). Göttingen: Wallstein.

Schmidt, Th. (1992). Pinscherseligkeit. Vom Elend des neuen deutschen Literaturstreits. In W. M. Lüdke \& D. Schmidt, Verkehrte Welten. Barock, Moral und schlechte Sitten (S. 171-189). Reinbek bei Hamburg: Rowohlt.

Schnell, R. (2003). Geschichte der deutschsprachigen Literatur seit 1945 (eine 2., überarb. und erw. Ausg.). Stuttgart: Metzler

Skare, R. \& Hoppe, R. B. (Hrsg.) (1999). Wendezeichen? Neue Sichtweisen auf die Literatur der DDR (= Amsterdamer Beiträge zur neueren Germanistik 46). (S. 15-43). Amsterdam: Rodopi.

Skare, R. (1999). 1989/90: Eine Wende in der deutschen Literaturgeschichte? Tendenzen der neueren Literaturgeschichtsschreibung. In R. Skare \& R. B. Hoppe (Hrsg.), Wendezeichen? Neue Sichtweisen auf die Literatur der DDR (= Amsterdamer Beiträge zur neueren Germanistik 46). (S. 15-43). Amsterdam: Rodopi.

Weninger, R. (2004) Streitbare Literaten. Kontroversen und Eklats in der deutschen Literatur von Adorno bis Walser. München: Beck.

Žmegač, V., Škreb, Z. \& Sekulić, L. (1997). Kleine Geschichte der deutschen Literatur: von den Anfängen bis zur Gegenwart. Weinheim: Beltz Athenäum. 